\title{
FORÇANTES DINÂMICAS E TÉRMICAS ASSOCIADAS A UM CASO DE PRECIPITAÇÃO INTENSA SOBRE O RIO GRANDE DO SUL, BRASIL
}

\author{
DORNELES, Viviane Rodrigues - vivianerdorneles@gmail.com \\ Universidade Federal de Pelotas / UFPel \\ RIQUETTI, Nelva Bugoni - nelvariquetti@hotmail.com \\ Universidade Federal de Pelotas / UFPel \\ NUNES, André - andre.nunes@ufpel.edu.br \\ Universidade Federal de Pelotas / UFPel
}

\begin{abstract}
RESUMO: A análise dos casos de eventos extremos de precipitação, e o conhecimento dos processos que os ocasionaram, é fundamental para o planejamento das condições de risco e identificação dos impactos sobre a drenagem da área. Em vista disso, o presente trabalho tem como objetivo a análise do ambiente de grande escala que propiciou a ocorrência de um sistema convectivo de mesoescala sobre o Rio Grande do Sul (RS) entre 20 e 25 de setembro de 2015, quando chuvas intensas, vento e granizos atingiram as regiões sul e nordeste do RS. A análise sinótica foi realizada empregando dados de reanálise do MERRA-2 e imagens do satélite GOES-13. Os resultados indicam a influência de sistemas termo-orográficos, como a Baixa do Noroeste Argentino (intensificada pelo Jato de Baixos Níveis), e sistemas dinâmicos, como o cavado a barlavento em altos níveis.
\end{abstract}

PALAVRAS-ChAVe: Sistema Convectivo de Mesoescala, Baixa do Noroeste Argentino, Advecção de vorticidade.

DYNAMIC AND THERMAL FORCES ASSOCIATED WITH A CASE OF INTENSE PRECIPITATION ON RIO GRANDE DO SUL, BRAZIL

\begin{abstract}
The analysis of the extreme events of precipitation, and the knowledge of the processes that caused them, is fundamental for the planning of the risk conditions and identification of the impacts on the drainage of the area. The objective of this work is to analyze the large-scale environment that caused the occurrence of a mesoscale convective system Rio Grande do Sul (RS) between September 20 and 25, 2015, when heavy rains, wind and hailstones reached the southern and northeastern regions of RS. The synoptic analysis was performed by employing MERRA-2 reanalysis data and GOES13 satellite images. The results showed the influence of thermo-orographic systems, as Northwestern Argentinean Low (intensified by Low Level Jet), and dynamical systems, as an upstream upper level through
\end{abstract}

KEYWORDS: Mesoscale Convective System, Northwestern Argentinean Low, Advection of vorticity.

FORZANTES DINÁMICAS Y TÉRMICAS ASOCIADAS A UN CASO DE PRECIPITACIÓN INTENSA SOBRE EL RÍO GRANDE DEL SUR, BRASIL

RESUMEN: El análisis de los casos de eventos extremos de precipitación, y el conocimiento de los procesos que los ocasionaron, es fundamental para la planificación de las condiciones de riesgo e identificación de los impactos sobre el drenaje del área. En vista de ello, el presente trabajo tiene como objetivo el análisis del ambiente de gran escala que propició la ocurrencia de un sistema convectivo de mesoescala sobre Rio Grande do Sul (RS) entre el 20 y el 25 de septiembre de 2015, cuando lluvias intensas, viento y granizos alcanzaron las regiones sur y nordeste del RS. El análisis sinóptico fue realizado empleando datos de reanálisis del MERRA-2 e imágenes del satélite GOES-13. Los resultados indican la influencia de sistemas termo-orográficos, como la Baja del 
Noroeste Argentino (intensificada por el Jet de Bajos Niveles), y sistemas dinámicos, como el cavado a barlovento en altos niveles.

PALABRAS CLAVE: Sistema Convectivo de Mesoescala, Baja del Noroeste Argentino, Advección de vorticidad.

FORCES DYNAMIQUES ET THERMIQUES ASSOCIÉES À UN CAS DE PRÉCIPITATION INTENSE SUR RIO GRANDE DO SUL, BRÉSIL

RÉSUMÉ: L'analyse des événements extrêmes de précipitations et la connaissance des processus qui les ont provoquées sont fondamentales pour la planification des conditions de risque et l'identification des impacts sur le drainage de la région. L'objectif de ce travail est d'analyser l'environnement à grande échelle qui a conduit à l'apparition d'un système convectif de moyenne échelle sur le Rio Grande do Sul (RS) entre le 20 et le 25 septembre 2015, lorsque les fortes pluies, le vent et les grêlons ont atteint les régions du sud et du nord-est de la RS. L'analyse synoptique a été réalisée à l'aide des données de réanalyse MERRA-2 et des images satellites GOES-13. Les résultats indiquent l'influence de systèmes thermo-orographiques, tels que le nord-ouest argentin inférieur (intensifié par le jet à basse altitude), et de systèmes dynamiques, tels que le creusé au vent à haute altitude.

MOTS-CLÉS: Système convectif méso-échelle, Nord-ouest argentin, Advection of vorticity.

\section{INTRODUÇÃO}

Eventos extremos ao atingirem certa localidade podem causar impactos sobre o meio em que estes ocorrem, sendo capaz de gerar vários transtornos sociais, ambientais e econômicos. Dentre esses eventos, a precipitação intensa tem despertado interesse devido às suas consequências, como alagamentos, desmoronamentos de terra, quedas de árvores, queda de energia, entre outros (CARMO e ANAZAWA, 2014), sendo os mais frequentes as enchentes/inundações, em especial ocorridos em centros urbanos (ALEXANDER, 2004). Dada a sua importância, são vários os estudos sobre eventos de precipitação intensa em diferentes regiões do Brasil, como por exemplo Silveira e Sartori (2010), Da Silva e Nunes (2011), Machado et al. (2012), Loureiro et al. (2014), Reboita et al. (2017a), Coutinho et al. (2017), entre outros.

O crescente registro de eventos extremos, sobretudo na Região Sul e Sudeste do Brasil, propõe que, estudar os sistemas atmosféricos que os coadjuvam é de ampla importância, não somente para a área da meteorologia, como também para a sociedade afetada (MENDONÇA, 2006). Neste caso, a compreensão e a análise dos sistemas que contribuem para a formação de um evento extremo auxiliam no melhoramento da previsão do tempo e na emissão precisa de alertas, por meio de um entendimento mais completo dos fenômenos meteorológicos, contribuindo para a prevenção da população.

A Região Sul do Brasil, sobretudo, apresenta um volume regular de precipitação ao longo do ano, decorrente da contribuição de vários sistemas que favorecem a ocorrência de chuvas (RIQUETTI et al., 2018). Dentre esses, destaca-se principalmente as frentes frias que atingem esta região (BORSATO e MENDONÇA, 2015), as quais propiciam a ocorrência de tempo severo.

Sistemas atmosféricos recorrentes nesta região, como os Sistemas Convectivos de Mesoescala (SCM), tem uma expressiva contribuição aos casos extremos e motivam volumes elevados de precipitação (SALIO et al., 2007). 
Dentre eles, pode-se citar as linhas de instabilidade (LI) que são zonas de instabilidade na qual uma série de tempestades estão dispostas de forma alinhada (VASCONCELLOS e CAVALCANTI, 2010), e os Complexos Convectivos de Mesoescala (CCM) sendo sistemas responsáveis por fortes chuvas e rajadas de vento (VELASCO e FRITSCH, 1987; VIANA et al., 2009; DURKEE e MOTE, 2010).

Estes sistemas atuam na mesoescala, ou seja, fenômenos atmosféricos menores que a escala sinótica. Porém são intensamente influenciados por forçantes de escala sinótica, tal como a Alta da Bolívia que é um sistema de alta pressão em altos níveis que ocorre sobre a região próxima a Bolívia. Esse sistema é associado com intenso aquecimento superficial, fluxos de calor sensível e latente e intensa precipitação (FIGUEROA et al., 1995; NUNES, 2017).

Outro sistema, que pode ser citado, em associação aos sistemas de altos níveis, relacionado a Alta da Bolívia e o aquecimento superficial, é a Baixa do Chaco. A Baixa do Chaco é um sistema de baixa pressão em superfície localizado na planície do Chaco próximo ao Paraguai, Bolívia e norte da Argentina, típico das estações mais quentes devido ao aquecimento da superfície e do escoamento de norte em baixos níveis (SAULO et al., 2004). Frequentemente este sistema é confundido com a Baixa do Noroeste Argentino, associada à sistemas transientes, situada mais ao sul e ocorre em todas as estações (SELUCHI e SAULO, 2012). Contudo, ambas são depressões de origem térmica, explicada pelo aquecimento em baixos níveis (SELUCHI et al., 2003). Desta forma, tem-se que um sistema precipitante pode ser influenciado tanto por forçantes térmicas, de baixos níveis, como advecção de temperatura e umidade, quanto forçantes dinâmicas, que são as de altos níveis, como Advecção de Vorticidade Ciclônica (AVC) e Corrente de Jato.

No geral, considerando a atuação de todos os sistemas meteorológicos que resultam em precipitação, a estação preponderante com relação às ocorrências de eventos de chuva intensa no RS é o inverno, conforme Nunes e Pereira (2017). Comportamento diferente, isto é, eventos extremos mais frequentes no verão, são observados no leste de Santa Catarina (NUNES e DA SILVA, 2013).

Com relação a previsão de sistemas que provocam precipitação, é sabido que estes estão diretamente relacionados às instabilidades decorrentes dos centros de baixa pressão, observados em baixos níveis. Desta forma, é comum, na meteorologia operacional, aplicar-se a análise aos campos superficial, principalmente ao campo de pressão ao nível médio do mar (pnmm). Entretanto, analisa-se também os níveis superiores, aqueles acima de $500 \mathrm{hPa}$, dado que nestes níveis pode estar indicada a fase de desenvolvimento do ciclo de vida de um sistema de baixos níveis. Sistemas de pressão em altos níveis são de mais fácil identificação, uma vez que se utiliza campos de linha de corrente, dado que em grandes altitudes o escoamento torna-se paralelo as isóbaras, ou seja, torna-se geostrófico, e também pelo campo de altura geopotencial dada a relação direta entre esta variável e a pressão atmosférica devido à aproximação hidrostática (NUNES, 2017).

Os fenômenos meteorológicos apresentam um histórico extenso de danos causados na região Sul do Brasil. Portanto, estudar as causas e consequências destes fenômenos sobre o estado é importante para diminuir os prejuízos à 
sociedade. Neste trabalho analisou-se um evento extremo, ocorrido entre 20 e 25 de setembro de 2015, quando chuvas intensas, vento e granizos atingiram grande parte das cidades do Rio Grande do Sul (RS), provocando transtornos de várias magnitudes.

Dentre os registros dos eventos, pode-se citar o que ocorreu na região Sul do RS, onde choveu em torno de $180 \mathrm{~mm}$ entre os dias 23 e 24 de acordo com as estações da Agência Nacional de Águas (ANA), acompanhado de rajadas de vento, que chegaram a $76 \mathrm{~km} \mathrm{~h}-1$ de acordo com as estações do Instituto Nacional de Meteorologia (INMET). Rios na região ficaram em observação de alerta por chance de extravasamento. Na região nordeste do Estado, os transtornos decorrentes de chuvas intensas, granizo e vento foram graves. Foi registrado quedas de árvores na região e em estradas de acesso onde carros que trafegavam foram atingidos, havendo interrompimento.

Para se determinar a previsibilidade das ferramentas utilizadas por meteorologistas previsores é de interesse que eventos extremos como este, que causam grandes impactos sociais e econômicos à sociedade, sejam estudados. Em vista disso, o presente trabalho tem como objetivo a análise do ambiente de grande escala associado ao evento de 20 a 25 de setembro de 2015, onde chuvas intensas, vento e granizos atingiram grande parte das cidades do RS.

\section{MATERIAIS E MÉTODOS}

A análise sinótica de todo o evento foi realizada por meio da observação de campos meteorológicos gerados de dados de reanálise, no caso, a reanálise do Modern Era Retrospective-Analysis for Research and Applications - Version 2 (MERRA-2) (GELARO et al., 2017). Os dados têm resolução espacial de $0.66^{\circ}$ de longitude e $0.5^{\circ}$ de latitude com intervalo de tempo de 6 horas. Os campos de pressão ao nível médio do mar, altura geopotencial em $500 \mathrm{hPa}$, direção e magnitude das componentes zonal e meridional do vento em 850 e $250 \mathrm{hPa}$, temperatura do ar em $850 \mathrm{hPa}$ e umidade específica em $850 \mathrm{hPa}$ foram visualizados por meio do pacote Grads $®$.

A nebulosidade associada ao evento foi analisada por meio das imagens do satélite Geostationary Operational Environmental Satellites (GOES-13), no canal infravermelho realçado, obtidas da Divisão de Satélites e Sistemas Ambientais do Instituto Nacional de Pesquisas Espaciais (DSA/INPE).

\section{RESULTADOS E DISCUSSÃO}

O caso em estudo apresentou eventos extremos de precipitação e presença de ventos fortes durante toda a semana dos dias 20 a 25 de setembro de 2015, que atingiu seu ápice entre o dia 24 e 25 de setembro, período para o qual concentram-se as análises, os resultados e discussões.

As imagens de satélite (Figura 1), referentes ao dia 24 mostram a nebulosidade associada a um Sistema Convectivo de Mesoescala (SCM) sobre grande parte do RS, sobretudo para região centro sul, deslocando-se de oeste para leste a partir das 06 UTC. 

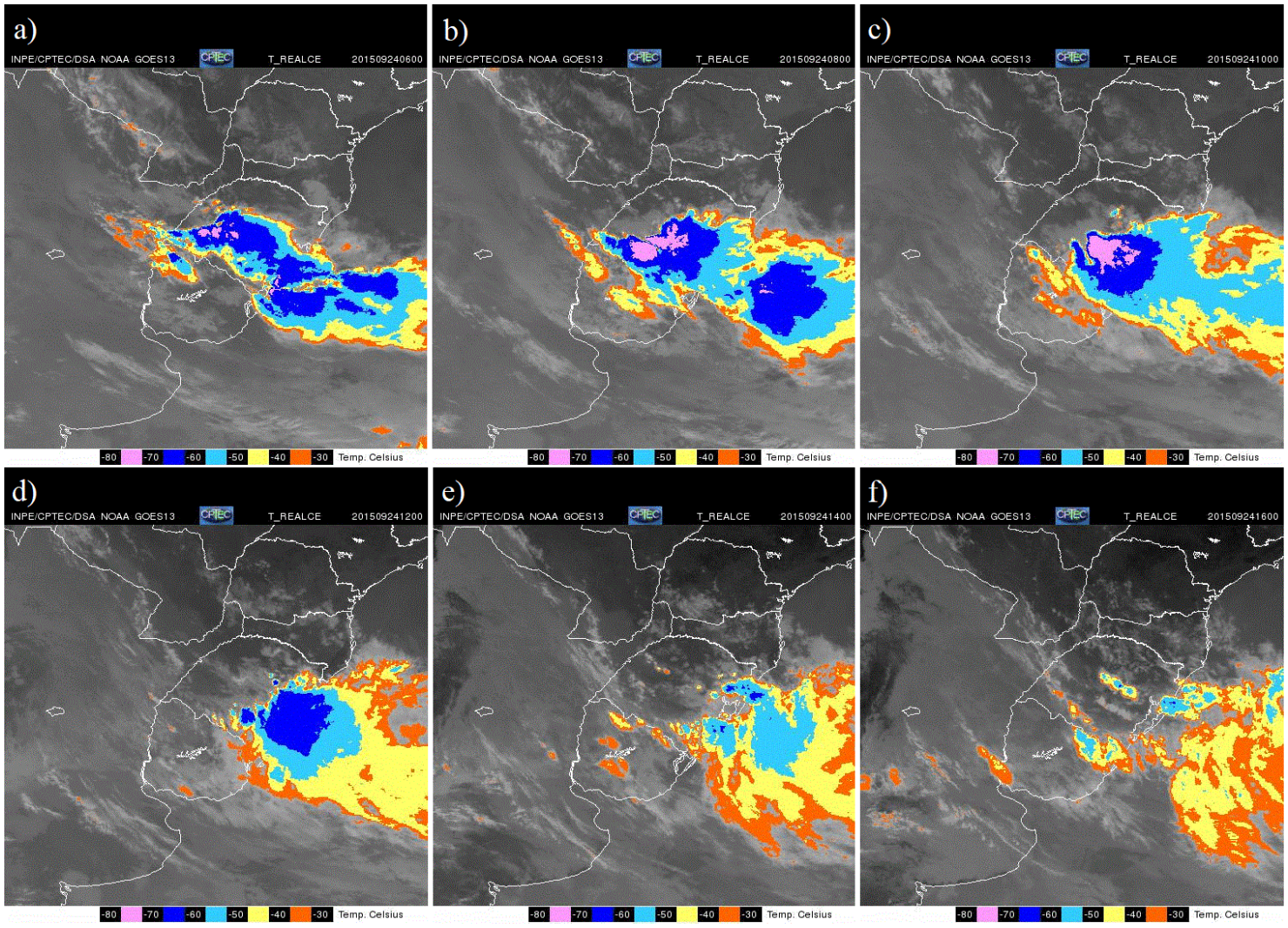

Figura 1 - Imagem do satélite GOES-13 no canal infravermelho (realçada) do dia 24 de setembro de 2015 às a) 06 UTC, b) 08 UTC, c) 10 UTC, d) 12 UTC, e) 14 UTC e f) 16 UTC.

A Figura 2 apresenta o campo de pnmm desde o dia 24 até as 06 UTC do dia 25 de setembro de 2015. Observa-se a Baixa do Noroeste Argentino (BNOA) (SELUCHI et al., 2003; ESCOBAR e SELUCHI, 2012) se estendendo até o RS, durante todo o período, ocasionando o fechamento mais claro de um centro de baixa pressão sobre o Estado às 06 UTC do dia 25 (Figura 2f). Este cavado estendido no sentido NO-SE indica a permanente chance de instabilidade em superfície e, consequentemente, precipitação intensa.

O presente caso é similar aos observados em Dorneles et al. (2017), que analisou 18 eventos extremos ocorridos no município de Pelotas/RS no período de 1982 a 2015, sendo que a maioria, 12 eventos, apresentou um padrão bem definido de centro de baixa pressão, iniciando-se como Baixa do Chaco e terminando como ciclone extratropical na costa do RS.

Também é similar ao de Da Silva e Wollmann (2016), que estudaram a influência dos sistemas atmosféricos envolvidos na ocorrência de desastres naturais na Quarta Colônia, localizada na região central do RS, e concluíram que os fenômenos de precipitação extrema, inundações, vendavais, granizo e tornado estiveram fortemente relacionados, dentre outros sistemas, com a Baixa do Chaco em conjunto com Massa Tropical Continental. 


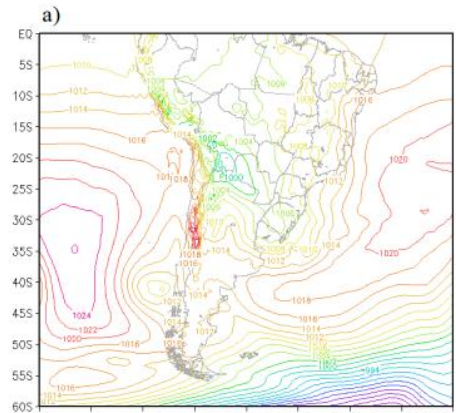

b)
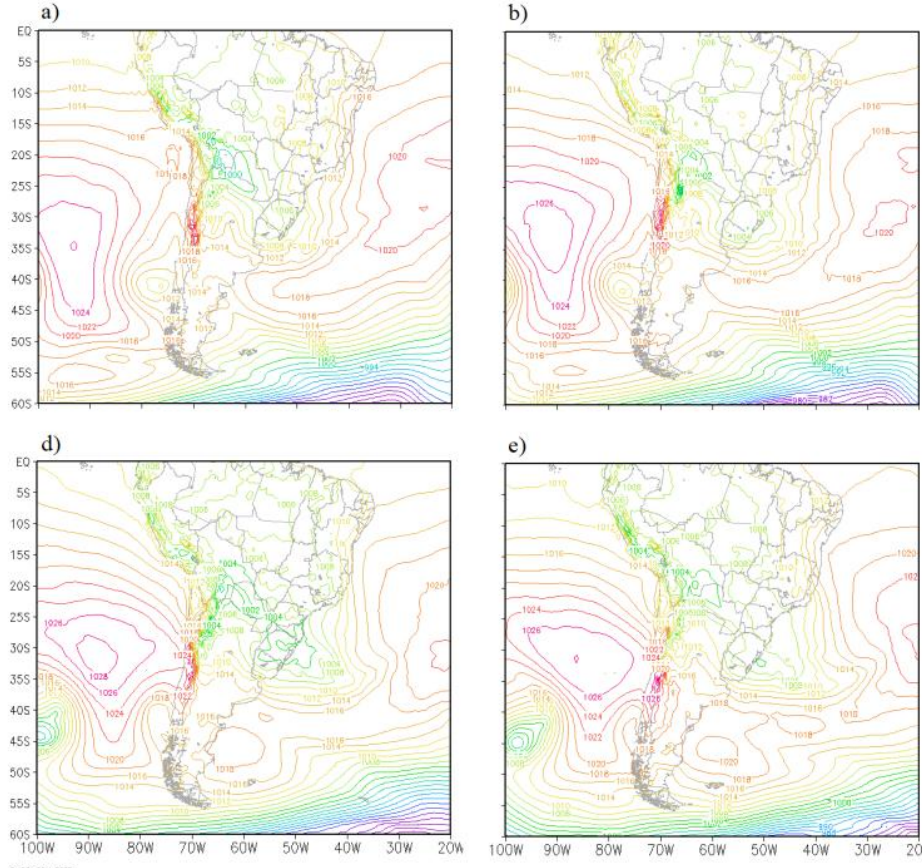

e)

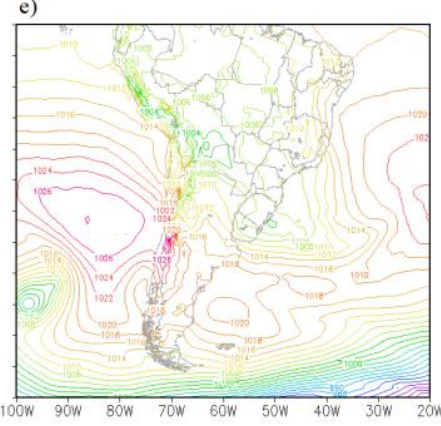

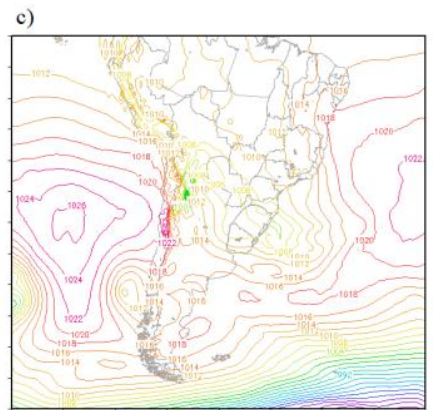

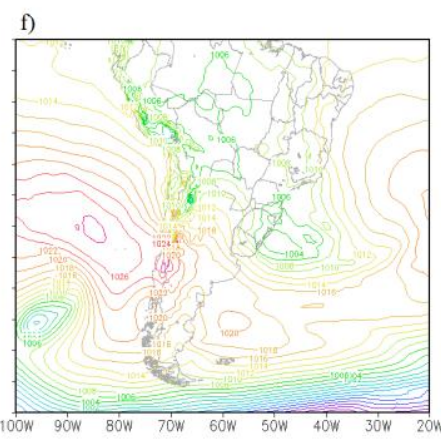

Figura 2 - Campo de pnmm (hPa) sendo a) 24/09/2015 às 00 UTC, b) 24/09/2015 às 06 UTC, c) 24/09/2015 às 12 UTC, d) 24/09/2015 às 18 UTC, e) 25/09/2015 às 00 UTC e f) 25/09/2015 às 06 UTC.

Os campos de pnmm e espessura da camada entre 500 e $1000 \mathrm{hPa}$ do período mais intenso do sistema (dia 24 e início do dia 25), são apresentados na Figura 3. Observa-se uma maior espessura (entre 5700 e $5800 \mathrm{mgp}$ ) sobre o RS se estendendo em direção ao Atlântico, indicando uma camada mais quente e, portanto, mais instável. A região de menor espessura à oeste, somado ao forte gradiente na região, indica a presença de advecções quente e fria, fundamentais para a formação de ciclones extratropicais (HOLTON, 2004). 

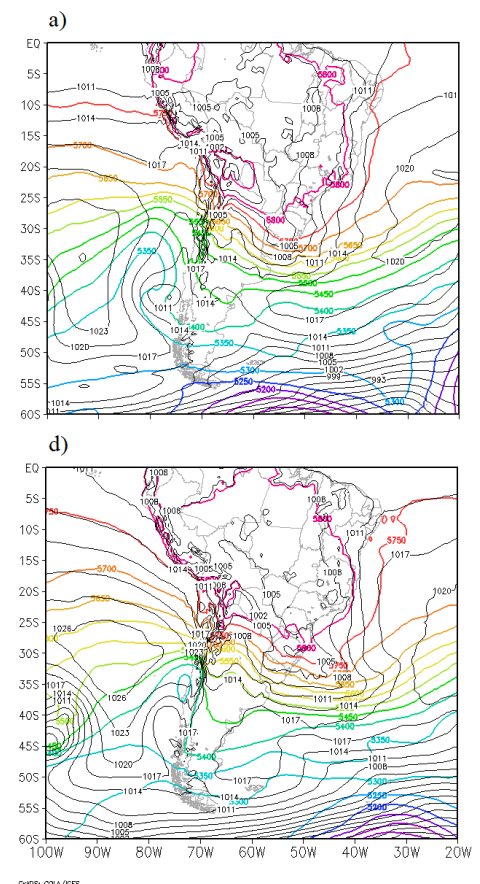

b)

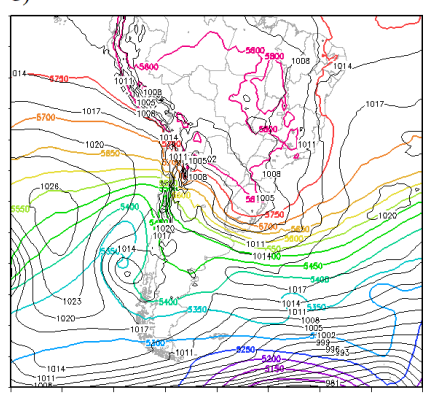

e)

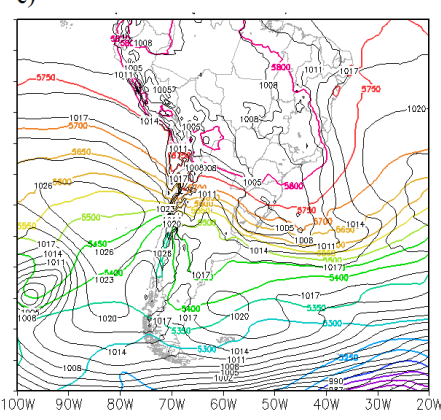

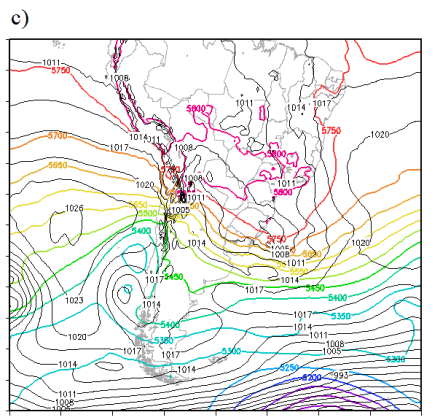

f)

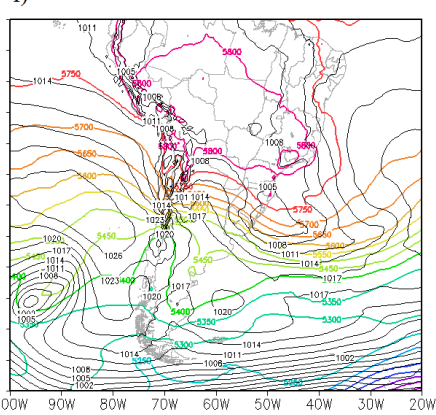

Figura 3 - Pnmm (linhas pretas, em hPa) e espessura da camada entre 500 e $1000 \mathrm{hPa}$ (linhas coloridas, em mgp) sendo a) 24/09/2015 às 00 UTC, b) 24/09/2015 às 06 UTC, c) $24 / 09 / 2015$ às 12 UTC, d) 24/09/2015 às 18 UTC, e) 25/09/2015 às 00 UTC e f) $25 / 09 / 2015$ às 06 UTC.

Observa-se no campo de altura geopotencial em 500 hPa no dia 24 às 18 UTC, a presença do cavado sobre a Argentina indicando Advecção de Vorticidade Ciclônica (AVC) sobre o RS o que, conforme a teoria quase-geostrófica, potencializa a chance de instabilidade em superfície (HOLTON, 2004).

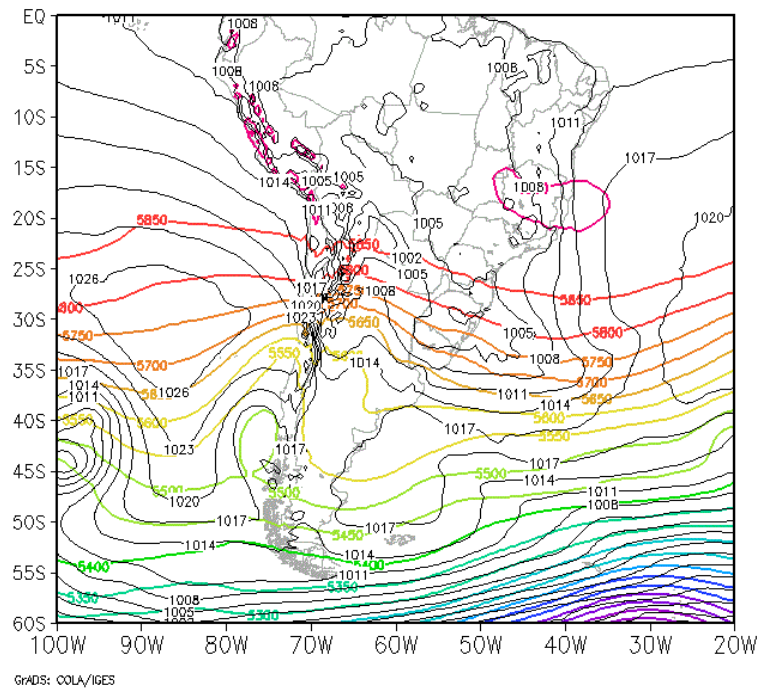

Figura 4 - Pnmm (linhas pretas, em hPa) e altura geopotencial em 500 hPa (linhas coloridas, em mgp) do dia 24 de setembro de 2015 às 18 UTC. 
A Figura 5 apresenta o campo de pnmm, linha de corrente e magnitude do vento em $250 \mathrm{hPa}$ do dia 24 de setembro de 2015 às 18 UTC. Observa-se a presença de núcleo da corrente de jato sobre RS e Uruguai, indicando regiões baroclínicas em superfície com chance de instabilidade. Tal núcleo fortalece a AVC, pois se encontra à sotavento do cavado em altos níveis. Como esperado, o escoamento em altos níveis explica a trajetória do SCM mostrada nas imagens de satélite.

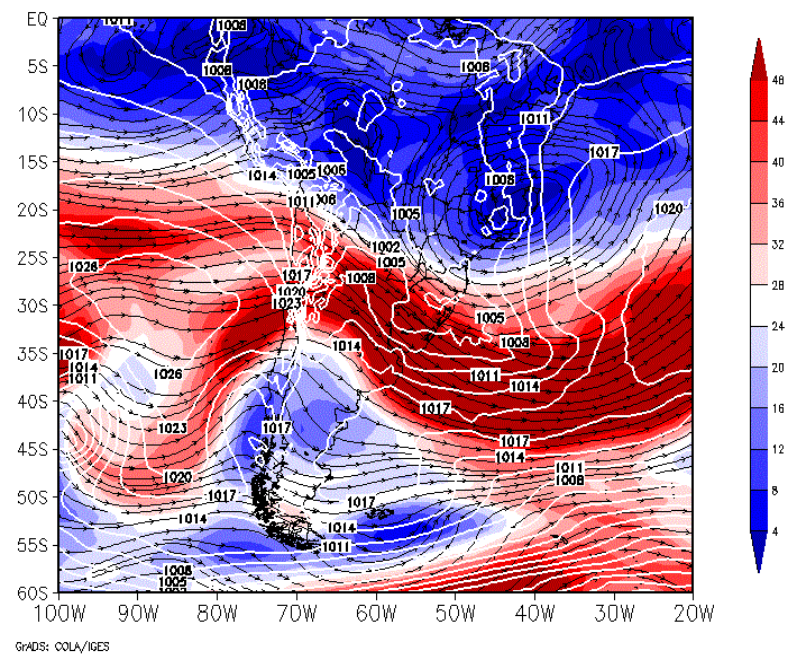

Figura 5 - Pnmm (linhas brancas, em hPa) e linha de corrente (linhas pretas) em 250 $\mathrm{hPa}$ e a magnitude do vento (sombreado, em m s-1) do dia 24 de setembro de 2015 às 18 UTC.

Observa-se um escoamento de norte, indicando transporte de calor e umidade da Amazônia para alimentar a instabilidade sobre o RS (Figura 6). Apesar de a magnitude do escoamento ultrapassar $12 \mathrm{~m} \mathrm{~s}-1$, os demais critérios para identificação do Jato de Baixos Níveis (especialmente o cisalhamento vertical), conforme Salio et al. (2002), não foram encontrados. Contudo, dada a intensidade e direção do escoamento, deve-se considerar o transporte de calor e umidade de baixas latitudes para a região de estudo.

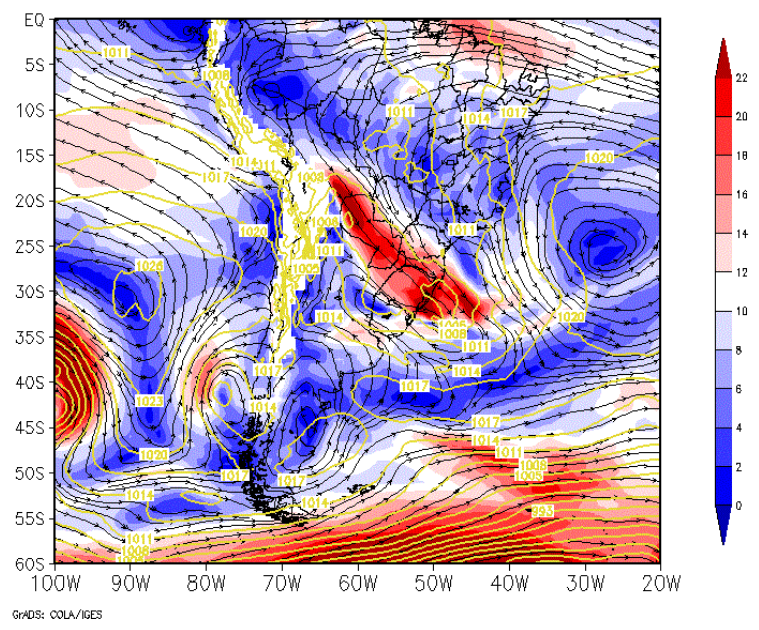

Figura 6 - Pnmm (linhas amarelas, em hPa), linha de corrente (linhas pretas) e magnitude do vento (sombreado, em m s-1) em 850 hPa do dia 24/09/2015 às 12 UTC. 
No nível de $850 \mathrm{hPa}$ pode-se observar a presença de umidade do ar sem sofrer tanta influência das condições naturais da superfície. No campo gerado para o evento observa-se que sempre há presença de umidade sobre a região, atingindo máximos valores no dia 2418 UTC (Figura 7a). Posteriormente, a umidade sobre o RS foi diminuindo até o dia 25/09 às 12 UTC (Figura 7b).

Ao se comparar a Figura 7 com a Figura 6, nota-se o esperado transporte de umidade de norte (Satyamurty et al., 1998; Bezerra e Nunes, 2017), o que está sempre associado a instabilidades sobre a região sul do Brasil (GRIMM, 2009).

a)

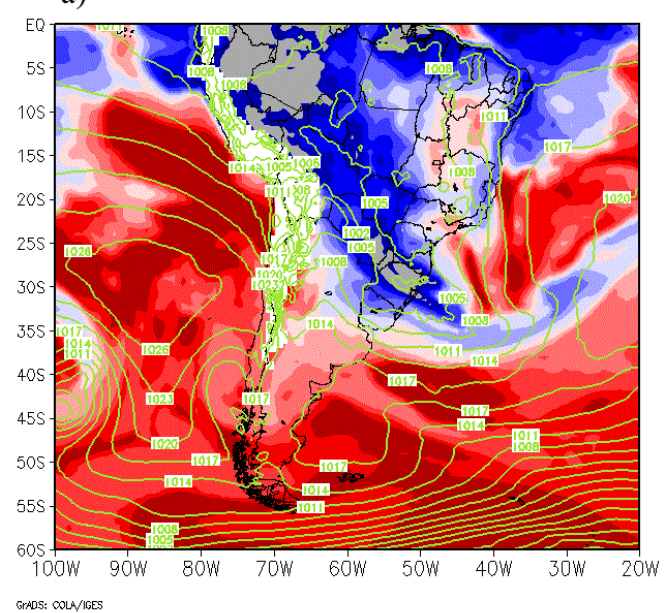

b)

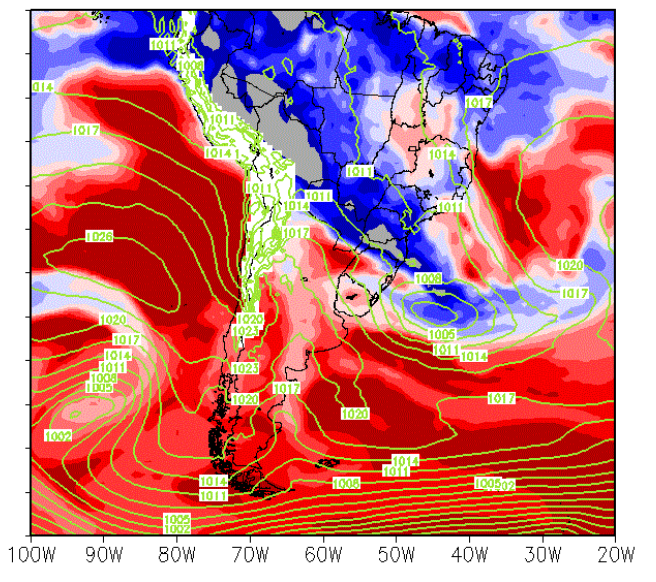

Figura 7 - Pnmm (linhas verdes, em hPa) e umidade específica em $850 \mathrm{hPa}$ (sombreado, em g kg-1) sendo a) dia 24/09/2015 às 18 UTC e b) 25/09/2015 às 12 UTC.

Observou-se que a temperatura em $850 \mathrm{hPa}$ permaneceu sempre com valores maiores que a redondeza (Figura 8) até dia 25/09 às 12 UTC (Figura $8 \mathrm{e})$, indicando a presença de massa tropical, que frequentemente está associada com instabilidade na região (GRIMM, 2009). Comparando a Figura 8 com as Figuras 3 e 6, identifica-se a advecção quente de norte, um fator ciclogenético associado à instabilidade (HOLTON, 2004; REBOITA et al., 2017b). 

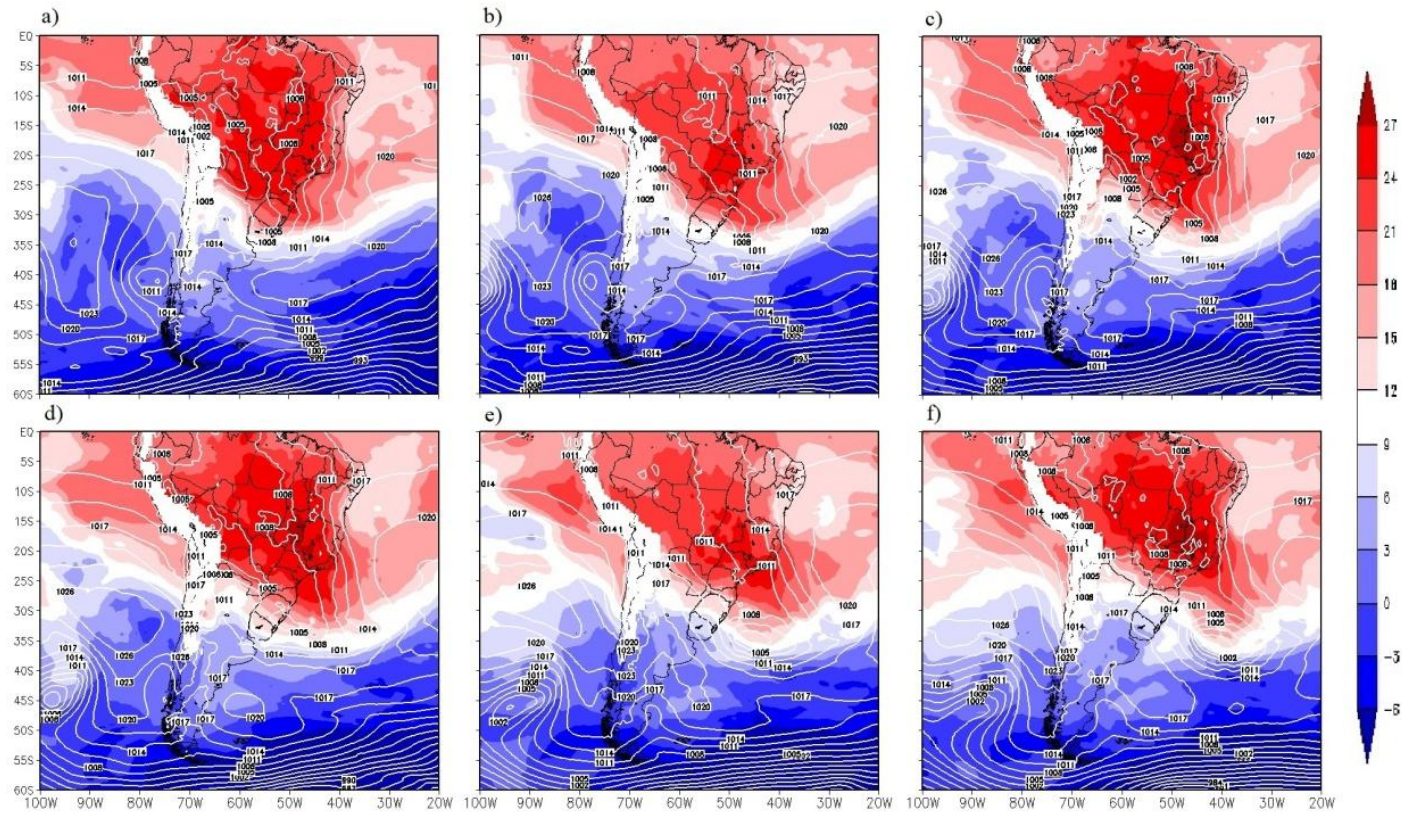

Figura 8 - PNMM (linhas brancas) e temperatura em $850 \mathrm{hPa}$ (sombreado, em ${ }^{\circ} \mathrm{C}$ ) do dia 24 e 25 de setembro de 2015 em que a) 24/09/2015 às 00 UTC, b) 24/09/2015 às 12 UTC, c) $24 / 09 / 2015$ às 18 UTC, d) 25/09/2015 às 00 UTC, e) 25/09/2015 às 12 UTC e f) 25/09/2015 às 18 UTC.

Por fim, com base no exposto, analisando-se os parâmetros estudados, menciona-se que o SCM que acarretou o evento de chuva intensa foi provocado por forçantes térmicas, oriundas de baixos níveis, e forçantes dinâmicas, proveniente de altos níveis.

\section{CONCLUSÕES}

A análise do ambiente de grande escala, associado ao evento de 20 a 25 de setembro de 2015, onde chuvas intensas, vento e granizos atingiram grande parte das cidades do RS, foi realizada. As imagens de satélite mostraram a presença de um SCM sobre grande parte do RS, especialmente entre 24 e 25 de setembro de 2015. Tal fenômeno acarretou em grandes volumes pluviométricos nas regiões sul e nordeste do RS. Fatores térmicos, como o escoamento de norte em $850 \mathrm{hPa}$ e a formação da Baixa do Noroeste Argentino, bem como fatores dinâmicos como o cavado em altos níveis, foram observados no desenvolvimento do SCM.

A extensão da BNOA, e consequentemente um escoamento de norte, quando observada pela magnitude de vento, propiciou o transporte de calor e umidade da Amazônia promovendo a chance instabilidade e precipitação intensa. Do mesmo modo, observou-se a presença de advecção de vorticidade ciclônica sobre o RS, que indica instabilidade em superfície, e a presença da corrente de jato sobre RS e Uruguai, indicando regiões baroclínicas. Como esperado, o presente trabalho mostrou que um conjunto de forçantes influenciou no desenvolvimento do sistema precipitante que acarretou em transtornos no RS em setembro de 2015. 


\section{REFERÊNCIAS BIBLIOGRÁFICAS}

ALEXANDER, D.E. Natural hazard on an unquiet earth. In: Unifying Geography. London: Routledge-Taylor and Francis Group, 2004, p. 266-268.

BEZERRA, J.M., NUNES, A.B. Anomalias de Circulação Atmosférica em Baixos Níveis do Verão (2013-2014) Anomalamente Quente nas Regiões Sul e Sudeste do Brasil, Anuário do Instituto de Geociências, Rio de Janeiro, UFRJ, v.40, n.1, p. 93-101, 2017.

BORSATO, V. A., MENDONÇA, F. A. Participação da Massa Polar Atlântica na dinâmica dos sistemas atmosféricos no Centro Sul do Brasil, Mercator, Fortaleza, v.14, n.1, p.113-130, 2015.

CARMO, R.L., ANAZAWA, T.M. Mortalidade por desastres no Brasil: o que mostram os dados, Ciência \& Saúde Coletiva, v.19, n.9, p.3669-3681, 2014.

COUTINHO, M.D.L.; COSTA, M.S.; GOMES, A.C.S.; MORAIS, M.D.C.; JACINTO, L.V.; LIMA, K.C.; SAKAMOTO, M.S. Estudo de caso: Evento extremo no Estado do Ceará entre os dias 03 e 04 de janeiro de 2015, Revista Brasileira de Climatologia. v. 20, p. 182-198, 2017.

DA SILVA, G.C.; NUNES, A.B. Análise dos eventos extremos de precipitação no leste de Santa Catarina: Estudo de Tendência, Ciência e Natura. Edição Especial. p. 251-254, 2011.

DA SILVA, G. C., WOLLMANN, C. A. Identificação e balanço de participação dos sistemas atmosféricos envolvidos na ocorrência de desastres naturais na Quarta Colônia - RS, no período de 1972 a 2014. XII Simpósio Brasileiro de Climatologia Geográfica. Variabilidade e Suscetibilidade Climática: Implicações ecossistêmicas e sociais 25 a 29 de outubro de 2016. Goiânia (GO)/UFG.

DORNELES, V. D., NUNES, A. B., CABALERO, C. B., OGASSAWARA, J. F., POSSA, T. M. Sistemas meteorológicos associados à eventos de precipitação intensa em Pelotas/RS. VII Encontro Sul Brasileiro de Meteorologia. 2017. Universidade Federal de Pelotas, UFPel, Pelotas (RS).

DURKEE, J. D., MOTE, T. L. A climatology of warm-season mesoscale convective complexes in subtropical South America. International Journal of Climatology. $\mathrm{v}$. 30, p.418-431, 2010.

ESCOBAR, G.C.J.; SELUCHI, M.E. Classificação sinótica dos campos de pressão atmosférica na América do Sul e sua relação com as baixas do Chaco e do noroeste argentino. Revista Brasileira de Meteorologia. v.27, n. 3, p. 365-375, 2012.

FIGUEROA, S.N., SATYAMURTY, P., SILVA DIAS, P.L. Simulations of the Summer Circulation over the South American Region with an Eta Coordinate Model, Journal of the Atmospheric Science, v.52, n.10, p.1573-1584, 1995.

GELARO, R.; MCCARTY, W.; SUÁREZ, M.J.; TODLING, R.; MOLOD, A.; TAKACS, L.; RANDLES, C.A.; DARMENOV, A.; BOSILOVICH, M.G.; REICHLE, R.; WARGAN, K.; COY, L.; CULLATHER, R.; DRAPER, C.; AKELLA, S.; BUCHARD, V.; CONATY, A.; SILVA, A.M.; GU, W.; KIM, G-K.; KOSTER, R.; LUCCHESI, R.; MERKOVA, D.; NIELSEN, J.E.; PARTYKA, G.; PAWSON, S.; PUTMAN, W.; RIENECKER, M.; SCHUBERT, S.D.; SIENKIEWICZ, M.; ZHAO, B. The Modern-Era Retrospective 
Analysis for Research and Applications, Version 2 (MERRA-2). Journal of Climate, v. 30, p. 5419-5454, 2017.

GRIMM, A.M. Clima da Região Sul do Brasil. in: CAVALCANTI, I.F.A.; FERREIRA, N.J.; JUSTI DA SILVA, M.G.A.; SILVA DIAS, M.A.F. (Org). Tempo e Clima no Brasil. ed. Oficina de Textos, São Paulo. pp. 259-275, 2009.

HOLTON, J.R. An Introduction to Dynamic Meteorology. Washington: Elsevier Academic Press, 2004. 553 p.

LOUREIRO, S. R., SARAIVA, M. J., SARAIRA, I., SENNA, C. R., FREDÓ, S. M. Estudo dos eventos extremos de precipitação ocorridos em 2009 no estado do Pará, Revista Brasileira de Meteorologia v.29, p.83-94, 2014.

MACHADO, C.C.C.; NÓBREGA, R.S.; OLIVEIRA, T.H.; ALVES, K.M.A.S. Distúrbio ondulatório de leste como condicionante a eventos extremos de precipitação em Pernambuco, Revista Brasileira de Climatologia. v. 11, p. 146-188, 2012.

MENDONÇA, F.A. Aquecimento global e suas manifestações regionais e locais: Alguns indicadores da Região Sul do Brasil, Revista Brasileira de Climatologia. v. 2, p. 71-86, 2006.

NUNES, A. B., PEREIRA, R. S. Estimativa de eventos de precipitação com potencial para alagamentos urbanos no Rio Grande do Sul. In: Nunes, A.B. E Mariano, G.L.(Org), Meteorologia em Tópicos - Volume 4. Pelotas: Clube de Autores, pp. 273-313, 2017.

NUNES, A.B. Case Study of Upper Tropospheric Meteorological Systems on South America: Synoptic Analysis, Anuário do Instituto de Geociências, Rio de Janeiro, UFRJ, v.40, p.70-82, 2017.

NUNES, A.B., DA SILVA, G.C. Climatology of extreme rainfall events in eastern and northern Santa Catarina State, Brazil: Present and future climate, Revista Brasileira de Geofísica, v.31, n.3, p.413-425, 2013.

REBOITA, M.S.; MARIETTO, D.M.G.; SOUZA, A.; BARBOSA, M. Caracterização atmosférica quando da ocorrência de eventos extremos de chuva na Região Sul de Minas Gerais, Revista Brasileira de Climatologia, v. 21, p. 20-37, 2017a.

REBOITA, M. S.; GAN, M. A.; ROCHA, R. P.; CUSTÓDIO, I.S. Ciclones em superfície nas latitudes austrais: Parte I - Revisão Bibliográfica, Revista Brasileira de Meteorologia, v. 32, n. 2, p. 171-186, 2017b.

RIQUETTI, N.B., DORNELES, V.R., NUNES, A.B. Estudo do caso de precipitação intensa sobre Foz do Iguaçu-PR em setembro de 2015, Revista Brasileira de Climatologia, v.22, p. 589-606, 2018.

SALIO, P., NICOLINI, M., SAULO, A.C. Chaco low-level Jet events characterization during the austral summer season, Journal of Geophysical Research, v.107, D24, 4816, 2002.

SALIO, P., NICOLINI, M., ZIPSER, E. J. Mesoscale convective systems over South America and their relationship with the South American low-level jet, Monthly Weather Review, v.135, p.1290-1309, 2007.

SAULO, A.C., FERREIRA, L., MEJIA, J., SELUCHI, M.E. Description of the thermal low characteristics using SALLJEX special observations, Clivar Exchanges, v.9, p.9-10, 2004. 
SELUCHI, M. E., SAULO, A. C. Baixa do Noroeste Argentino e Baixa do Chaco: Características, diferenças e semelhanças, Revista Brasileira de Meteorologia, v. 27 , n. 1 , p. $49-60,2012$

SILVEIRA, R.D.; SARTORI, M.G.B. Relação entre tipos de tempo, eventos de precipitação extrema e inundações no espaço urbano de São Sepé-RS, Revista Brasileira de Climatologia, v. 7, p.63-82, 2010.

VASCONCELLOS, F. C., CAVALCANTI, I. F. A. Uma avaliação das previsões do modelo regional Eta em alta resolução para dois casos de chuva intensa ocorridos na região da Serra do mar, Revista Brasileira de Meteorologia, v.25, n.4, p.501-512, 2010.

VELASCO, I., FRITSCH, J.M. Mesoscale convective complexes in the Americas, Journal of the Geophysical Research, v.92, p.9591-9613, 1987.

VERA, C.S., BAEZ, J., DOUGLAS, M., EMMANUEL, C.B., MARENGO, J.A., MEITIN, J., NICOLINI, M., NOGUES-PEAGLE, J., PEAGLE, J., PENALBA, O., SALIO, P., SAULO, C., SILVA DIAS, M.A., SILVA DIAS, P., ZIPSER, E. The South American low-Level Jet Experiment, Bulletin of American Meteorological Society, v.87, p.63-77, 2006.

VERNEKAR, A.D., KIRTMAN, B.P., FENNESSY, M.J. Low-level jet and their effects on the South American summer climate as simulated by the NCEP Eta model, Journal of Climate, v.16, p.297-311, 2003.

VIANA, D. R., AQUINO, F. E., BURGOBRAGA, R., FERREIRA, N. J. Mesoscale convective complexes in Rio Grande do Sul between October and December of 2003 and associated precipitation, Revista Brasileira de Meteorologia, v.24, n.3, p.276-291, 2009 